\title{
Hadron Structure from the Feynman-Hellmann Theorem
}

A. J. Chambers ${ }^{*},{ }^{a}$ J. Dragos, ${ }^{a b}$ R. Horsley, ${ }^{c}$ Y. Nakamura,${ }^{d}$ H. Perlt, ${ }^{e}$ D. Pleiter, ${ }^{f g}$ P. E. L. Rakow, ${ }^{h}$ G. Schierholz, ${ }^{i}$ A. Schiller, ${ }^{e}$ K. Somfleth, ${ }^{a}$ H. Stüben, ${ }^{j}$ R. D. Young ${ }^{a k}$ and J. M. Zanotti ${ }^{a}$

${ }^{a}$ CSSM, Department of Physics, University of Adelaide, Adelaide SA 5005, Australia

${ }^{b}$ Michigan

${ }^{c}$ School of Physics and Astronomy, University of Edinburgh, Edinburgh EH9 3JZ, UK

${ }^{d}$ RIKEN Advanced Institute for Computational Science, Kobe, Hyogo 650-0047, Japan

${ }^{e}$ Institut für Theoretische Physik, Universität Leipzig, 04103 Leipzig, Germany

${ }^{f}$ JSC, Jülich Research Centre, 52425 Jülich, Germany

${ }^{g}$ Institut für Theoretische Physik, Universität Regensburg, 93040 Regensburg, Germany

${ }^{h}$ Theoretical Physics Division, Department of Mathematical Sciences University of Liverpool, Liverpool L69 3BX, UK

${ }^{i}$ Deutsches Elektronen-Synchrotron DESY, 22603 Hamburg, Germany

${ }^{j}$ Regionales Rechenzentrum, Universität Hamburg, 20146 Hamburg, Germany

${ }^{k}$ CoEPP, Department of Physics, University of Adelaide, Adelaide SA 5005, Australia

E-mail: alexander. chambers@adelaide.edu.au

\begin{abstract}
The determination of hadronic form factors at large momentum transfers has been a challenging problem in lattice QCD simulations. Here we show how the Feynman-Hellmann method may be extended to non-forward matrix elements to calculate hadronic form factors in lattice QCD at much higher momenta than previously accessible. We are able to determine the electromagnetic form factors of the pion and nucleon up to approximately $6 \mathrm{GeV}^{2}$, with results for $G_{E} / G_{M}$ in the proton agreeing well with experimental results.
\end{abstract}

34th annual International Symposium on Lattice Field Theory

24-30 July 2016

University of Southampton, UK

\footnotetext{
${ }^{*}$ Speaker.
} 


\section{Introduction}

One of the great challenges of hadron physics is to build consistent pictures of the internal structures of strongly-interacting particles. An important aspect of this endeavour is the calculation of electromagnetic form factors, which describe the distribution of electromagnetic currents in hadrons.

Recoil polarisation experiments at Jefferson Lab show that the ratio of the electric and magnetic form factors in the nucleon, $\mu_{p} G_{E p} / G_{M p}$, decreases approximately linearly for $Q^{2} \gtrsim 0.5 \mathrm{GeV}^{2}$ (see e.g. [1, 2, 3, 4]). Experimental results at high-momentum scales are not yet precise enough to determine whether this trend continues and there is a zero crossing. Resolving the scaling of the form factors in this domain is one of the key physics goals of the upgraded CEBAF at Jefferson Lab.

The large- $Q^{2}$ behaviour of the pion electromagnetic form factor $F_{\pi}$ is also challenging to investigate experimentally (see $[5,6,7]$ for recent innovative advances). This information is important for understanding the transition from the soft to the hard regime in QCD (see [8] for a recent example). At present, the experimental data is not able to reliably discriminate different models describing the transition to the asymptotic domain [9].

Lattice calculations of hadronic form factors have typically focussed on the study of processes at low-momentum transfer (see e.g. $[10,11,12,13,14,15]$ ), with only limited studies at large $Q^{2} \gtrsim 3 \mathrm{GeV}^{2}[16,17]$. These calculations are difficult because the signals fall with $Q^{2}$, and the signal-to-noise ratio deteriorates. This also makes it difficult to evaluate the degree of excited-state contamination $[16,18,14,19,20]$.

In this work we demonstrate how high-momentum transfer in hadron form factors may be accessed on the lattice using an extension of the Feynman-Hellmann theorem to non-forward matrix elements. This builds upon the techniques developed for forward matrix elements [21, 22, 23, 24] (see also [25, 26, 27, 28, 29, 30, 31, 32] for similar related techniques). These methods allow one to access matrix elements from 2-point correlators, rather than a more complicated analysis of 3-point functions, which simplifies the elimination of excited-state contamination. The calculations are performed with Breit frame kinematics $\left(E\left(\vec{p}^{\prime}\right)=E(\vec{p})\right)$ and hence one maximises the momentum transfer for any given state momentum, reducing the noise in the correlation function.

\section{Feynman-Hellmann Methods}

The Feynman-Hellmann method for the calculation of forward matrix elements is described in [22]. Here we describe only the subtleties involved in extending the technique to non-forward matrix elements. Suppose the QCD Lagrangian is modified in a lattice simulation such that

$$
\mathscr{L}(y) \rightarrow \mathscr{L}(y)+\lambda\left(e^{+i \vec{q} \cdot \vec{y}}+e^{-i \vec{q} \cdot \vec{y}}\right) \mathscr{O}(y),
$$

where $\mathscr{O}$ is a quark-bilinear operator and $\lambda$ is a freely-varying real parameter. It may be shown that the shift in the energy of a hadron state $H\left(\vec{p}^{\prime}\right)$ resulting from a shift in $\lambda$ from $\lambda=0$ is proportional to a matrix element of the operator $\mathscr{O}$,

$$
\left.\frac{\partial E_{H}\left(\vec{p}^{\prime}\right)}{\partial \lambda}\right|_{\lambda=0}=\frac{\left\langle H\left(\vec{p}^{\prime}\right)|\mathscr{O}(0)| H(\vec{p})\right\rangle}{\left\langle H\left(\vec{p}^{\prime}\right) \mid H\left(\vec{p}^{\prime}\right)\right\rangle}
$$


where $\vec{p}=\vec{p}^{\prime} \pm \vec{q}$. In order for this result to hold, $\vec{p}$ and $\vec{p}^{\prime}$ must satisfy the Breit frame condition $E_{H}(\vec{p})=E_{H}\left(\vec{p}^{\prime}\right)$. States not satisfying this requirement do not receive energy shifts at $\mathscr{O}(\lambda)$.

Following this procedure, we may calculate non-forward matrix elements for any particular operator $\mathscr{O}$ by performing hadron spectroscopy for multiple values of $\lambda \neq 0$. Connected quark contributions are calculated by inverting quark propagators according to the modified action corresponding to Eq. (2.1). Determining disconnected contributions requires the generation of new gauge ensembles [24].

\section{Simulation Details}

In this work, we use an ensemble of 1700 gauge field configurations with $2+1$ flavours of non-perturbatively $O(a)$-improved Wilson fermions and a lattice volume of $L^{3} \times T=32^{3} \times 64$. The lattice spacing $a=0.074(2) \mathrm{fm}$ is set using a number of singlet quantities [33, 34, 35, 36]. The clover action used comprises the tree-level Symanzik improved gluon action together with a stout smeared fermion action, modified for the implementation of the FH method [22]. The hopping parameters $\left(\kappa_{l}, \kappa_{s}\right)=(0.120900,0.120900)$ correspond to a pion mass of $\sim 470 \mathrm{MeV}$. To study electromagnetic form factors, quark propagators are calculated with the modified Lagrangian

$$
\mathscr{L}(y) \rightarrow \mathscr{L}(y)+\left(e^{+i \vec{q} \cdot \vec{y}}+e^{-i \vec{q} \cdot \vec{y}}\right) \bar{q}(y) \lambda \cdot \gamma q(y),
$$

for multiple values of $\vec{q}$, where either $\lambda_{2}$ or $\lambda_{4}$ take non-zero values of $1 \times 10^{-4}$ or $-1 \times 10^{-5}$. Note that we only use Breit-frame kinematics with $\vec{p}^{\prime}=-\vec{p}$. This choice allows us to minimise $\vec{p}^{2}$ for each value of $\vec{q}^{2}$, and hence minimise the noise in the correlator. This choice of kinematics also results in nucleon energy shifts that are directly proportional to $G_{E}$ and $G_{M}$.

\section{Results}

\subsection{Electromagnetic Form Factors of the Nucleon}

Individual quark flavour contributions to the Euclidean decompositon of the vector current matrix element of the nucleon are written in terms of the Dirac and Pauli $\left(F_{1}^{q}\right.$ and $\left.F_{2}^{q}\right)$ form factors,

$$
\left\langle N\left(p^{\prime}, s^{\prime}\right)\left|\bar{q}(0) \gamma_{\mu} q(0)\right| N(p, s)\right\rangle=\bar{u}\left(p^{\prime}, s^{\prime}\right)\left[\gamma_{\mu} F_{1}^{q}\left(Q^{2}\right)+\sigma_{\mu v} \frac{q_{v}}{2 M_{N}} F_{2}^{q}\left(Q^{2}\right)\right] u(p, s),
$$

where we denote the invariant 4-momentum transfer squared as $Q^{2}=-q^{2}=-\left(p^{\prime}-p\right)^{2}$. The Sachs electromagnetic form factors are defined by

$$
G_{E}^{q}=F_{1}^{q}-\frac{Q^{2}}{(2 M)^{2}} F_{2}^{q}, \quad G_{M}^{q}=F_{1}^{q}+F_{2}^{q} .
$$

For Breit frame kinematics where $\vec{p}^{\prime}=-\vec{p}$, the energy shifts resulting from insertion of the temporal and spatial components of the current are proportional to the electric and magnetic form factors respectively,

$$
\left.\frac{\partial E_{N}}{\partial \lambda_{4}}\right|_{\lambda=0} \stackrel{\vec{p}^{\prime}=-\vec{p}}{=} \frac{M_{N}}{E_{N}} G_{E}^{q},\left.\quad \frac{\partial E_{N}}{\partial \lambda_{i}}\right|_{\lambda=0} \stackrel{\vec{p}^{\prime}=-\vec{p}}{=} \frac{[\hat{e} \times \vec{q}]_{i}}{2 E_{N}} G_{M}^{q} .
$$


Here $\hat{\vec{e}}$ is the spin polarisation vector determined by the choice of polarisation direction of the nucleon.

Fig. 1 shows results for the proton electric and magnetic form factors neglecting disconnected contributions. In the low- $Q^{2}$ region we compare with results computed on the same ensembles using a variationally-improved 3-point function approach. Good agreement is observed in the region of comparable $Q^{2}$. The Feynman-Hellmann approach is seen to extract a clean signal to much higher momentum transfers than have previously been accessible. Fig. 2 displays the extraction
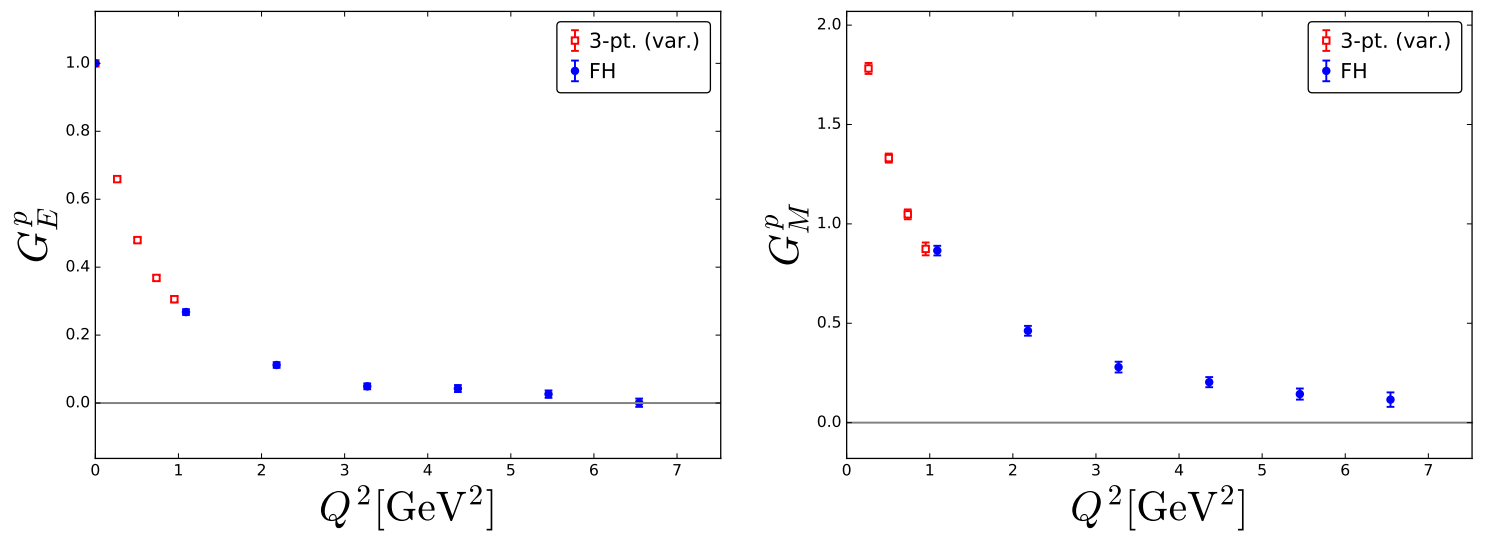

Figure 1: $G_{E}$ and $G_{M}$ for the proton from the Feynman-Hellmann method and from a variational analysis of three-point functions on the same ensemble (as described in [20]).

of the ratio $G_{E} / G_{M}$ as a function of $Q^{2}$ from the Feynman-Hellmann technique, a variational approach and experimental data. The overall trend is seen to compare very well with the experimental data.

\subsection{Electromagnetic Form Factor of the Pion}

Individual quark flavour contributions to the pion form factor are defined by

$$
\left\langle\pi\left(p^{\prime}\right)\left|\bar{q}(0) \gamma_{\mu} q(0)\right| \pi(p, s)\right\rangle=\frac{\left[p+p^{\prime}\right]_{\mu}}{2 E_{\pi}} F_{\pi}^{q}\left(Q^{2}\right)
$$

With the modified fermion action, pion energy shifts are given by

$$
\left.\frac{\partial E_{\pi}}{\partial \lambda_{4}}\right|_{\lambda=0} \stackrel{\vec{p}^{\prime}=-\vec{p}}{=} F_{\pi}^{q},\left.\quad \frac{\partial E_{\pi}}{\partial \lambda_{i}}\right|_{\lambda=0} \stackrel{\vec{p}^{\prime}=-\vec{p}}{=} 0 .
$$

Following a similar analysis as that for the nucleon, we show the determination of the pion form factor in Fig. 2, along with a comparison to experimental data. The signal-to-noise ratio achieved gives confidence that future lattice simulations will be able to provide important insight into the transition between the perturbative and nonperturbative regimes.

\section{Conclusion}

In this work we have extended the Feynman-Hellmann technique to access non-forward matrix elements. We demonstrate that application of the technique provides a dramatic improvement in 

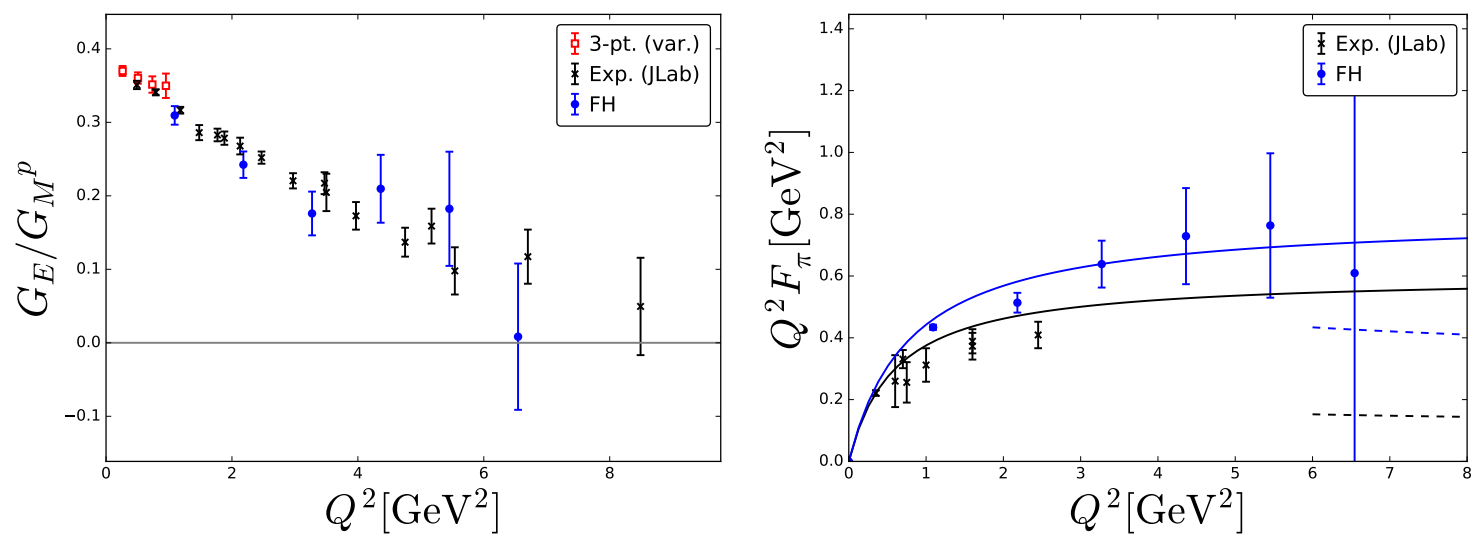

Figure 2: On the left, the ratio $G_{E} / G_{M}$ for the proton from application of the Feynman-Hellmann method, from a variational analysis of three-point functions, and from experiment [37, 4, 3]. On the right, the scaled pion form factor $Q^{2} F_{\pi}$ from the Feynman-Hellmann technique and from experiment [7]. The solid lines are the vector meson dominance at the relevant pion masses, and the dotted lines are the asymptotic values predicted by perturbative QCD (see [8] for a discussion of this value and its limitations).

the ability to extract nucleon and pion form factors at high momentum transfers. An additional improvement that we intend to pursue is the use of improved operators that couple more strongly to boosted hadron states, as proposed in [38].

\section{Acknowledgments}

The numerical configuration generation was performed using the BQCD lattice QCD program [39], on the IBM BlueGeneQ using DIRAC 2 resources (EPCC, Edinburgh, UK), the BlueGene P and Q at NIC (Jülich, Germany) and the Cray XC30 at HLRN (Berlin-Hannover, Germany). Some of the simulations were undertaken using resources awarded at the NCI National Facility in Canberra, Australia, and the iVEC facilities at the Pawsey Supercomputing Centre. These resources are provided through the National Computational Merit Allocation Scheme and the University of Adelaide Partner Share supported by the Australian Government. This work was supported in part through supercomputing resources provided by the Phoenix HPC service at the University of Adelaide. The BlueGene codes were optimised using Bagel [40]. The Chroma software library [41], was used in the data analysis. This investigation has been supported by the Australian Research Council under grants FT120100821, FT100100005 and DP140103067 (RDY and JMZ). HP was supported by DFG grant SCHI 422/10-1.

\section{References}

[1] M. K. Jones et al., $G(E(p)) / G(M(p))$ ratio by polarization transfer in polarized e $p-\& g t ; e$ polarized p, Phys. Rev. Lett. 84, 1398 (2000), [nucl-ex/9910005].

[2] O. Gayou et al., Measurement of $G(E p) / G(M p)$ in polarized-e $p-\& g t$; e polarized-p to $Q^{2}=5.6$ $G e V^{2}$, Phys. Rev. Lett. 88, 092301 (2002), [nucl-ex/0111010]. 
[3] V. Punjabi et al., Proton elastic form-factor ratios to $Q^{2}=3.5 \mathrm{GeV}^{2}$ by polarization transfer, Phys. Rev. C71, 055202 (2005), [nucl-ex/0501018].

[4] A. J. R. Puckett et al., Final Analysis of Proton Form Factor Ratio Data at $Q^{2}=4.0,4.8$ and 5.6 GeV², Phys. Rev. C85, 045203 (2012), [1102.5737].

[5] J. Volmer et al., Measurement of the Charged Pion Electromagnetic Form-Factor, Phys. Rev. Lett. 86, 1713 (2001), [nucl-ex/0010009].

[6] T. Horn et al., Determination of the Charged Pion Form Factor at $Q^{2}=1.60$ and $2.45 \mathrm{GeV} / \mathrm{c}^{2}$, Phys. Rev. Lett. 97, 192001 (2006), [nucl-ex/0607005].

[7] G. M. Huber et al., Charged pion form-factor between $Q^{2}=0.60 \mathrm{GeV}^{2}$ and $2.45 \mathrm{GeV}^{2}$. II. Determination of, and results for, the pion form-factor, Phys. Rev. C78, 045203 (2008), [0809.3052].

[8] L. Chang, I. C. Cloët, C. D. Roberts, S. M. Schmidt, and P. C. Tandy, Pion electromagnetic form factor at spacelike momenta, Phys. Rev. Lett. 111, 141802 (2013), [1307.0026].

[9] T. Horn and C. D. Roberts, The pion: an enigma within the Standard Model, J. Phys. G43, 073001 (2016), [1602.04016].

[10] S. Collins et al., Dirac and Pauli form factors from lattice QCD, Phys. Rev. D84, 074507 (2011), [1106.3580].

[11] C. Alexandrou et al., Nucleon electromagnetic form factors in twisted mass lattice QCD, Phys. Rev. D83, 094502 (2011), [1102.2208].

[12] P. E. Shanahan et al., Magnetic form factors of the octet baryons from lattice QCD and chiral extrapolation, Phys. Rev. D89, 074511 (2014), [1401.5862].

[13] P. E. Shanahan et al., Electric form factors of the octet baryons from lattice QCD and chiral extrapolation, Phys. Rev. D90, 034502 (2014), [1403.1965].

[14] J. R. Green et al., Nucleon electromagnetic form factors from lattice QCD using a nearly physical pion mass, Phys. Rev. D90, 074507 (2014), [1404.4029].

[15] S. Capitani et al., Nucleon electromagnetic form factors in two-flavor QCD, Phys. Rev. D92, 054511 (2015), [1504.04628].

[16] H.-W. Lin, S. D. Cohen, R. G. Edwards, K. Orginos, and D. G. Richards, Lattice Calculations of Nucleon Electromagnetic Form Factors at Large Momentum Transfer, (2010), 1005.0799.

[17] J. Koponen, A. C. Zimermmane-Santos, C. T. H. Davies, G. P. Lepage, and A. T. Lytle, Prediction of the meson electromagnetic form factor at high $Q^{2}$ from full lattice QCD, (2017), 1701.04250.

[18] B. J. Owen et al., Variational Approach to the Calculation of gA, Phys. Lett. B723, 217 (2013), [1212.4668].

[19] B. Yoon et al., Controlling Excited-State Contamination in Nucleon Matrix Elements, Phys. Rev. D93, 114506 (2016), [1602.07737].

[20] J. Dragos et al., Nucleon matrix elements using the variational method in lattice QCD, Phys. Rev. D94, 074505 (2016), [1606.03195].

[21] R. Horsley et al., A Lattice Study of the Glue in the Nucleon, Phys. Lett. B714, 312 (2012), [1205.6410].

[22] A. J. Chambers et al., Feynman-Hellmann approach to the spin structure of hadrons, Phys. Rev. D90, 014510 (2014), [1405.3019]. 
[23] A. J. Chambers et al., A novel approach to nonperturbative renormalization of singlet and nonsinglet lattice operators, Phys. Lett. B740, 30 (2015), [1410.3078].

[24] A. J. Chambers et al., Disconnected contributions to the spin of the nucleon, Phys. Rev. D92, 114517 (2015), [1508.06856].

[25] W. Detmold, Flavor singlet physics in lattice QCD with background fields, Phys. Rev. D71, 054506 (2005), [hep-lat/0410011].

[26] M. Engelhardt, Neutron electric polarizability from unquenched lattice QCD using the background field approach, Phys. Rev. D76, 114502 (2007), [0706.3919].

[27] W. Detmold, B. C. Tiburzi, and A. Walker-Loud, Extracting Electric Polarizabilities from Lattice QCD, Phys. Rev. D79, 094505 (2009), [0904.1586].

[28] W. Detmold, B. C. Tiburzi, and A. Walker-Loud, Extracting Nucleon Magnetic Moments and Electric Polarizabilities from Lattice QCD in Background Electric Fields, Phys. Rev. D81, 054502 (2010), [1001.1131].

[29] T. Primer, W. Kamleh, D. Leinweber, and M. Burkardt, Magnetic properties of the nucleon in a uniform background field, Phys. Rev. D89, 034508 (2014), [1307.1509].

[30] W. Freeman, A. Alexandru, M. Lujan, and F. X. Lee, Sea quark contributions to the electric polarizability of hadrons, Phys. Rev. D90, 054507 (2014), [1407.2687].

[31] M. J. Savage et al., Proton-proton fusion and tritium $\beta$-decay from lattice quantum chromodynamics, (2016), 1610.04545.

[32] C. Bouchard, C. C. Chang, T. Kurth, K. Orginos, and A. Walker-Loud, On the Feynman-Hellmann Theorem in Quantum Field Theory and the Calculation of Matrix Elements, (2016), 1612.06963.

[33] R. Horsley et al., SU(3) flavour symmetry breaking and charmed states, PoS LATTICE2013, 249 (2013), [1311.5010].

[34] V. G. Bornyakov et al., Wilson flow and scale setting from lattice QCD, (2015), 1508.05916.

[35] W. Bietenholz et al., Tuning the strange quark mass in lattice simulations, Phys. Lett. B690, 436 (2010), [1003.1114].

[36] W. Bietenholz et al., Flavour blindness and patterns of flavour symmetry breaking in lattice simulations of up, down and strange quarks, Phys. Rev. D84, 054509 (2011), [1102.5300].

[37] A. J. R. Puckett et al., Recoil Polarization Measurements of the Proton Electromagnetic Form Factor Ratio to $Q^{2}=8.5 \mathrm{GeV}^{2}$, Phys. Rev. Lett. 104, 242301 (2010), [1005.3419].

[38] G. S. Bali, B. Lang, B. U. Musch, and A. Schäfer, Novel quark smearing for hadrons with high momenta in lattice QCD, Phys. Rev. D93, 094515 (2016), [1602.05525].

[39] Y. Nakamura and H. Stüben, BQCD - Berlin quantum chromodynamics program, $\mathrm{PoS}$ LATTICE2010, 040 (2010), [1011.0199].

[40] P. A. Boyle, The BAGEL assembler generation library, Comput. Phys. Commun. 180, 2739 (2009).

[41] R. G. Edwards and B. Joo, The Chroma software system for lattice QCD, Nucl. Phys. Proc. Suppl. 140, 832 (2005), [hep-lat/0409003]. 